\title{
Distribution of mineral and products of its etching in dentin-adhesive interface.
}

\author{
V.M.Dusevich, J.D.Eick
}

University of Missouri - Kansas City, Kansas City, MO, 64108

The application of dental cutting instruments creates a smear layer on top of dentin that consists of the debris of collagen and mineral (hydroxyapatite). For proper adhesion of modern dental adhesives, this layer and underlying dentin should be etched with acids to remove the mineral and expose the dentin collagen network. Adhesives penetrate the network creating a so-called hybrid layer and form a bond to dentin [1]. In a number of studies a problem of mineral distribution in the hybrid layer after etching was discussed, based mostly on the results of the observation of micrographs [2] and more recently, on such analytical methods as micro Raman spectroscopy [3]. Observation alone without analytical analysis is not sufficient, and the resolution of micro Raman spectroscopy was limited to 1.5 micrometers.

The high spatial resolution of EDS in STEM makes this method highly attractive for analysis of this interface. Dental bonding systems, ScotchBond Multipurpose Plus (SBM) and Prompt L-Pop (PLP) (both from 3M Espe, St. Paul, MN, USA), were each applied to 3 dentin specimens. One specimen from each group was air dried without any treatment. The remaining specimens were fixed with $2.5 \%$ glutaraldehyde and were dehydrated and embedded without post fixation with heavy metals. Ultra thin sections were cut from all specimens without decalcification. No staining was applied. The specimens were analyzed with a STEM CM12 and, after mounting grids on carbon stubs, with a field-emission SEM XL30 (FEI Company, Hillsboro, OR). Results obtained from air dried specimens and properly embedded specimens were similar, demonstrating that dehydration and embedding did not affect the non-demineralized dentin and hybrid layer. Five line scans at the magnification of 70,000 were obtained from each specimen. For both bonding systems, the width of the partially demineralized dentin layer was small: less than $50 \mathrm{~nm}$ (Fig. 1a) in 16 measurements, not greater than $100 \mathrm{~nm}$ but more than $50 \mathrm{~nm}$ in 6 measurements and in the other 8 the width was less than $200 \mathrm{~nm}$ but greater than $100 \mathrm{~nm}$ (Fig. 1b). Line scans taken at lower magnifications across the whole SBM hybrid layer and beyond it demonstrated that there was no presence of the mineral in the hybrid layer, but above it, in the remnants of smear layer, the presence of $\mathrm{Ca}$ (and no $\mathrm{P}$ ) was observed (Fig. 2). SBM bonding system used a separate acid etching step with washing afterwards, so while some by-products of etching (such as Ca-containing) could be trapped in the smear layer debris, others could be washed out. In contrast, PLP is a "single bottle" system, where etching agents are incorporated in adhesive resin. All etching products remained in the hybrid and adhesive layers, which showed higher concentrations of $\mathrm{Ca}$ and especially P (Fig. 3). Additional P may come from the etchant of the bonding system which contains phosphoric esters. Line scans also showed a higher intensity of $\mathrm{Ca}$ and $\mathrm{P}$ characteristic radiation in PLP resin above the hybrid layer. The lower intensity of $\mathrm{Ca}$ and $\mathrm{P}$ in the hybrid layer compared to the adhesive resin layer may be due to collagen absorbing around $50 \%$ of the volume of the hybrid layer and replacing the $\mathrm{Ca}$ and $\mathrm{P}$ containing resin. Blocks from which the sections were cut with the ultramicrotome were better specimens for the EDS analysis of bulk dentin specimens in the SEM, compared to other methods of specimen preparation, which lead to an uneven specimen surface (fracturing) or to excessive smear (polishing). The analysis of these blocks confirmed those of the STEM findings concerning the $\mathrm{Ca}$ and $\mathrm{P}$ distribution in the dentin-adhesive interface in which high spatial EDS resolution was not critical. Figure 4 
represents $\mathrm{Ca}$ and $\mathrm{P}$ distribution in the bulk PLP specimen and is similar to Figure 2.

References:

[1] J.D.Eick et al., Crit Rev Oral Biol Med, 8(3) (1997) 306-335

[2] B.Van Meerbeek et al., J Dent Res 72(2) (1993) 495-501

[3] Y.Wang, P.Spencer, J Biomed Mater Res 60 (2002) 300-308

[4] This project was supported in part by NIDCR research grant DE09696
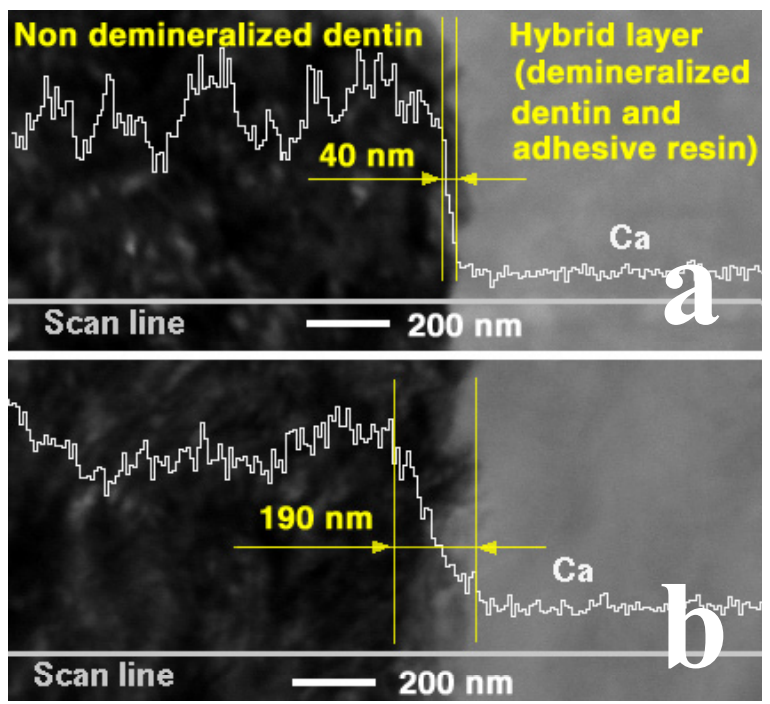

FIG. 1. Line scans of SBM specimens showing narrow (a) and wide (b) partially demineralized layers. STEM micrograph.

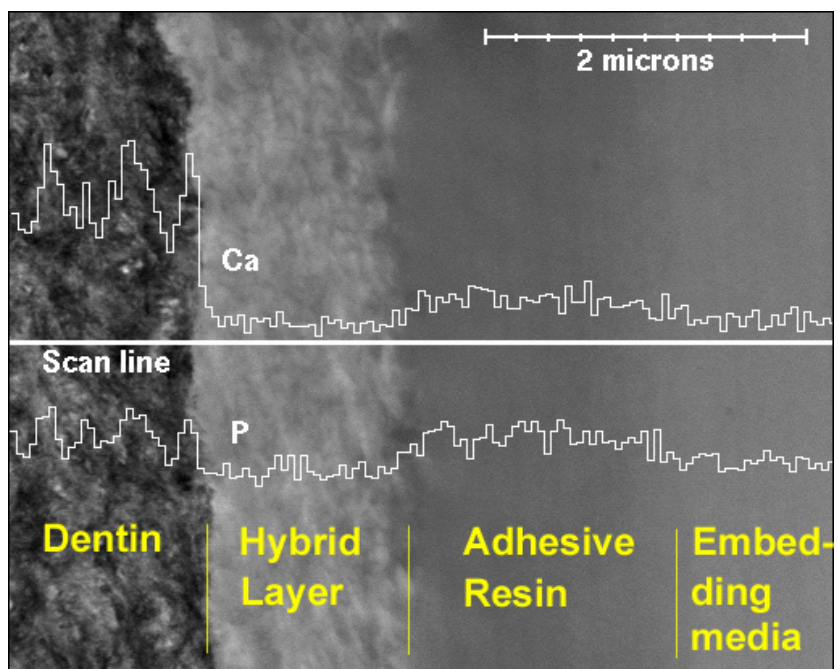

FIG. 3. Line scan of PLP specimen. STEM micrograph.
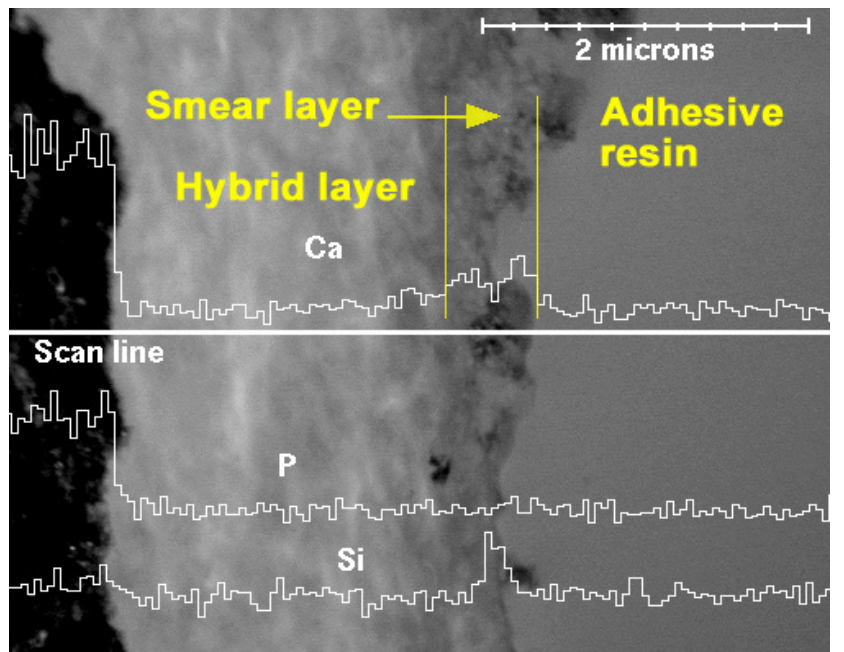

FIG. 2. Line scan of SBM specimen showing an increase of $\mathrm{Ca}$ concentration in the remnants of the smear layer. Silicon containing particles are from the etching gel. STEM micrograph.

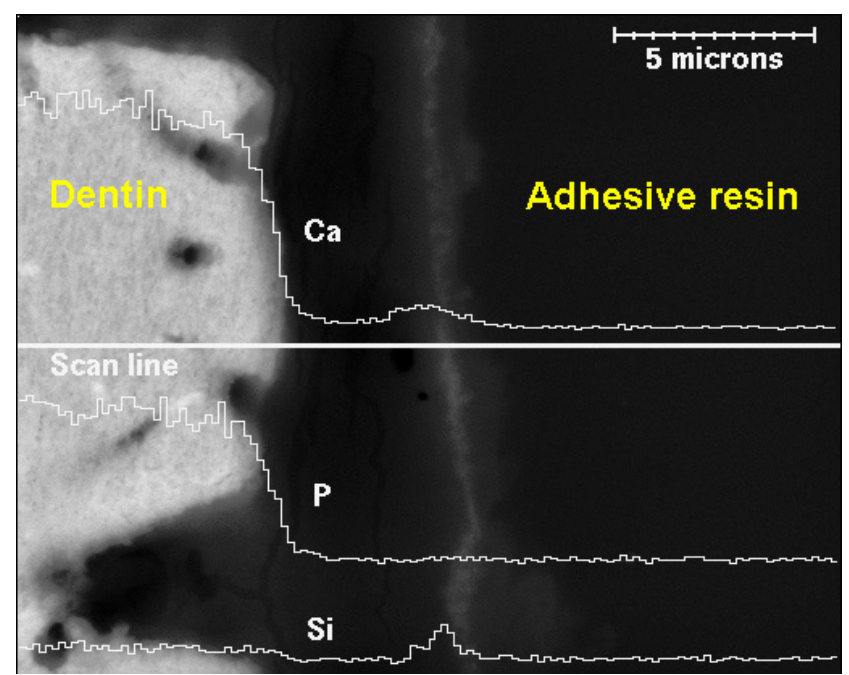

FIG. 4. Line scan of the bulk specimen used for cutting ultrathin sections, carbon coated. SBM bonding system, backscattered electrons detector. SEM micrograph. 